\title{
Nonlinear stability in the transport of intense bunched beams
}

Thales M. Corrêa da Silva, Felipe B. Rizzato, Renato Pakter, and Yan Levin

Citation: Physics of Plasmas 23, 113102 (2016); doi: 10.1063/1.4967708

View online: https://doi.org/10.1063/1.4967708

View Table of Contents: http://aip.scitation.org/toc/php/23/11

Published by the American Institute of Physics

\section{Articles you may be interested in}

Parametric sum envelope instability of periodically focused intense beams

Physics of Plasmas 23, 090705 (2016); 10.1063/1.4963851

Theory based scaling of edge turbulence and implications for the scrape-off layer width

Physics of Plasmas 23, 112502 (2016); 10.1063/1.4966564

Self-channelling of intense laser pulses in underdense plasma and stability analysis

Physics of Plasmas 23, 113101 (2016); 10.1063/1.4966560

Erratum: "Existence domains of electrostatic solitary structures in the solar wind plasma" [Phys. Plasmas 23, $062902(2016)]$

Physics of Plasmas 23, 119903 (2016); 10.1063/1.4967357

Collisionless electrostatic shock formation and ion acceleration in intense laser interactions with near critical density plasmas

Physics of Plasmas 23, 113103 (2016); 10.1063/1.4967946

A viable non-axisymmetric non-force-free field to represent solar active regions

Physics of Plasmas 23, 114504 (2016); 10.1063/1.4967759

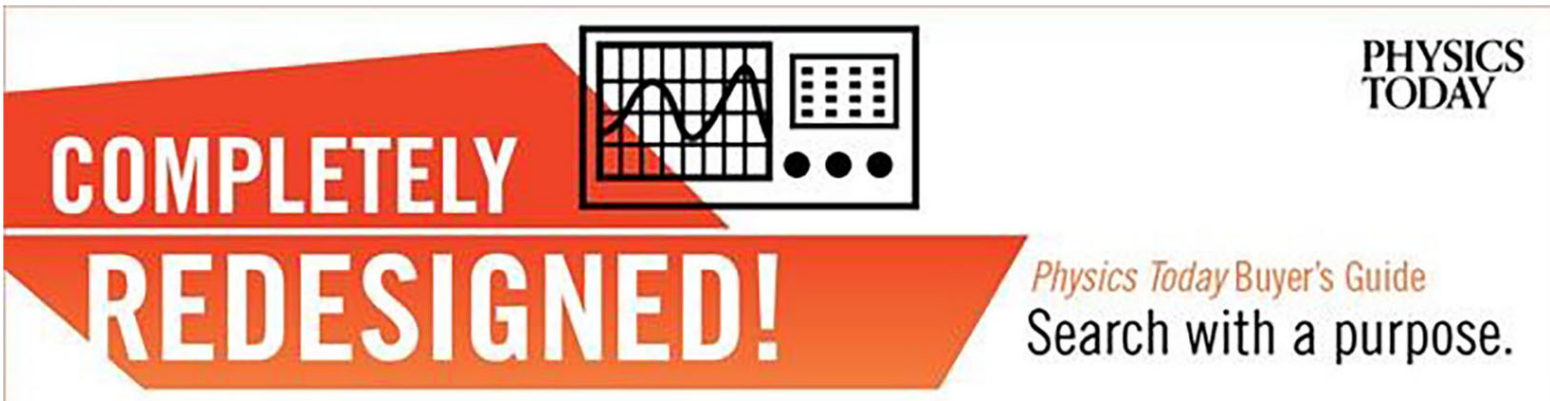




\title{
Nonlinear stability in the transport of intense bunched beams
}

\author{
Thales M. Corrêa da Silva, Felipe B. Rizzato, Renato Pakter, and Yan Levin \\ Instituto de Física, Universidade Federal do Rio Grande do Sul, Caixa Postal 15051, 91501-970 Porto Alegre, \\ RS, Brazil
}

(Received 17 August 2016; accepted 26 October 2016; published online 9 November 2016)

\begin{abstract}
The paper investigates the nonlinear coupling of envelope modes of oscillation for intense bunched beams. Initially, the analysis concentrates on the case of spherically symmetric beams for which longitudinal and transverse focusing forces are assumed to be the same. It is investigated how externally induced spherically symmetric breathing oscillations may nonlinearly drive the growth of ellipsoidal modes which can break the spherical beam symmetry. Next, a more general case in which the focusing forces are not symmetric such that the matched beam already presents an ellipsoidal shape is studied. It is found that depending on the parameters of the system, even a very small mismatch amplitude can drive an instability, which leads to an effective coupling of longitudinal and transversal envelope oscillations by means of the space-charge forces. Use is made of Poincaré plots and the stability index of periodic orbits to perform a detailed analysis of the location of the instability in the parameter space and how it affects the beam transport. Selfconsistent numerical simulations are performed in order to verify the onset of the nonlinear instability and its effect on the evolution of the RMS size and emittance of the beam. Published by AIP Publishing. [http://dx.doi.org/10.1063/1.4967708]
\end{abstract}

\section{INTRODUCTION}

Nonlinear dynamics effects play a major role in the transport of intense charged particle beams. This is so because for such systems the space charge forces are strong enough to couple the dynamics of different beam particles, leading to a possible onset of a very complex behavior. An example is the occurrence of nonlinear resonances and chaos driven by periodic focusing fields acting on the envelope oscillations of nearly matched beams. ${ }^{1-8}$

In many practical situations, however, the beam is not even close to the equilibrium (matched) condition. The mismatch is caused by the experimental difficulties in the beam launching process, by the intrinsic current oscillations in the system, ${ }^{9}$ or by design to minimize the extent of the beam halo. ${ }^{10}$ In such cases, the beam tends to relax to a stationary state along its trajectory. ${ }^{11}$ The length scale for the full relaxation to occur in the beam transport depends heavily on the specific situation but may take from a few to more than $10^{3}$ betatron oscillations. ${ }^{12,13}$ Again, the nonlinear effects play a major role, being the main mechanism leading to the relaxation. For instance, for initially RMS mismatched beams, the relaxation occurs as some of the particles are nonlinearly driven by the envelope oscillations to form a halo in a process similar to the evaporative cooling. ${ }^{12,14-25}$ Another example is when the initial particle distribution does not correspond to a stationary state and density waves appear in the system. The relaxation is then achieved through a nonlinear growth of the density wave amplitude which eventually leads to wave breaking. ${ }^{13,26-32}$

In both cases discussed above, the relaxation is accompanied by the emittance growth. However, depending on the time scales of the different phenomena, other nonlinear effects may become important much before there is any sizable emittance growth, and while the system is still far from the final stationary state. A particular example is the nonlinear coupling between breathing and quadrupole envelope modes for continuous beams. It has been shown that if the breathing oscillation amplitude exceeds a certain threshold, it drives the quadrupole mode unstable, breaking the initial axial symmetry of the particle distribution. ${ }^{33}$ The beam develops an elliptical shape with increase in its size along one direction, which may induce beam losses. Curiously, a similar behavior is found for self gravitating systems. ${ }^{34}$

In this paper, we extend the analysis of the nonlinear coupling of envelope modes to the case of bunched beams. Initially, we consider the case of spherically symmetric beams where longitudinal and transversal (with respect to the direction of propagation) focusing forces are assumed to be the same ${ }^{25}$ and investigate how spherically symmetric breathing oscillations may nonlinearly induce the growth of ellipsoidal modes which can break the beam symmetry. It is found that above a certain mismatch amplitude threshold, there is, indeed, such coupling with an effective exchange of energy between the modes. However, in comparison to the continuous beam case, it is found that for bunched beams this nonlinear instability only takes place for much larger initial mismatch. This happens because for spherical beams, the linear eigenfrequencies of symmetric and antisymmetric oscillations around the matched solution are far from being commensurate. Therefore, a large oscillating amplitude is necessary in order to lock the frequencies and generate a resonance that causes the instability. We next consider a more general case in which the focusing forces along the longitudinal and transverse directions are not the same so that the matched beam already presents an ellipsoidal shape. In this case, and depending on the parameters of the system, one may find a commensurate relation between the linear eigenfrequencies of oscillation which may facilitate the onset of 
instabilities. ${ }^{35}$ In fact, we find that for such parameters very small mismatch amplitudes are necessary to drive the frequency lock and the instability which causes an effective coupling between longitudinal and transversal envelope oscillations. Using Poincaré plots, ${ }^{36}$ we are able to identify the instability as connected to a period doubling bifurcation of a given orbit. From the Poincaré plots, we can also determine which trajectories are most affected by the instability. By calculating the stability index using a Newton-Raphson method, ${ }^{37}$ we construct parameter space plots showing in detail where the instability occurs. Finally, we use selfconsistent $\mathrm{N}$-body simulations in order to verify the onset of the nonlinear instability and its effect on the evolution of the RMS size and emittance of the beam.

\section{MODEL}

Let us consider a beam of nonrelativistic particles uniformly distributed in an ellipsoid of semi-axis $\rho_{m}$ and $z_{m}$. The center of the beam is propagating with a constant axial velocity $V_{b}$ along the $z$-axis of a laboratory frame. The beam particles are subject to an external linear focusing force which tends to balance the Coulomb self-repulsion. Although in real devices the focusing force generally varies along the transport channel, the nonlinear effects investigated here are expected to occur at a length scale that is long compared to the focusing force variation. Hence, we assume an average uniform focusing field-the so-called smooth beam approximation. The dynamics of each particle is governed by

$$
\begin{gathered}
\frac{d^{2} \boldsymbol{\rho}}{d s^{2}}=-k_{\rho}^{2} \boldsymbol{\rho}-\nabla_{\perp} \psi, \\
\frac{d^{2} \tilde{z}}{d s^{2}}=-k_{z}^{2} \tilde{z}-\frac{\partial \psi}{\partial \tilde{z}},
\end{gathered}
$$

where $s=V_{b} t$ is the beam central axis position that plays the role of a scaled time variable, $\boldsymbol{\rho}=(x, y)$ is the transverse displacement from the beam center, $\tilde{z}=z-s$ is the axial displacement, and $k_{\rho}$ and $k_{z}$ are the vacuum phase advances that measure the strength of the transverse and axial focusing force, respectively. In Eq. (1), $\psi$ is the self-field potential that satisfies the Poisson equation

$$
\nabla^{2} \psi=-\frac{4 \pi K}{N} n(\mathbf{r}, s),
$$

where $\mathbf{r}=(x, y, \tilde{z})$ is the displacement vector from the beam center, $n(\mathbf{r}, s)$ is the beam density, $K=q^{2} N / m V_{b}^{2}$ is a parameter that measures the beam intensity, and $N=\int n d \mathbf{r}$ is the constant number of particles in the bunch.

The bulk oscillations of the beam can be studied using envelope equations. The beam envelope is a measure of the beam size along a given direction. It is conveniently defined as $\rho_{b}=\left[5\left\langle\rho^{2}\right\rangle / 2\right]^{1 / 2}$, for the transverse envelope, and $z_{b}$ $=\left[5\left\langle\tilde{z}^{2}\right\rangle\right]^{1 / 2}$, for the longitudinal envelope, where $\langle\ldots\rangle$ $=(1 / N) \int \ldots n d \mathbf{r}$. The " $5 / 2$ " and "5" factors are introduced so that $\rho_{b}=\rho_{m}$ and $z_{b}=z_{m}$ at $s=0$ for a beam with a uniform density. Taking two derivatives of $\rho_{b}$ and $z_{b}$ with respect to $s$, using Eq. (1) and conveniently rearranging the terms, we can write

$$
\begin{gathered}
\frac{d^{2} \rho_{b}}{d s^{2}}=-k_{\rho}^{2} \rho_{b}-\frac{5}{2 \rho_{b}}\left\langle\boldsymbol{\rho} \cdot \nabla_{\perp} \psi\right\rangle+\frac{\epsilon_{\rho}^{2}}{\rho_{b}^{3}}, \\
\frac{d^{2} z_{b}}{d s^{2}}=-k_{z}^{2} z_{b}-\frac{5}{z_{b}}\left\langle\tilde{z} \frac{\partial \psi}{\partial \tilde{z}}\right\rangle+\frac{\epsilon_{z}^{2}}{z_{b}^{3}},
\end{gathered}
$$

where

$$
\begin{gathered}
\epsilon_{\rho}=\frac{5}{2}\left[\left\langle\boldsymbol{\rho}^{2}\right\rangle\left\langle\dot{\boldsymbol{\rho}}^{2}\right\rangle-\langle\boldsymbol{\rho} \cdot \dot{\boldsymbol{\rho}}\rangle^{2}\right]^{1 / 2}, \\
\epsilon_{z}=5\left[\left\langle\tilde{z}^{2}\right\rangle\left\langle\dot{\tilde{z}}^{2}\right\rangle-\langle\tilde{z} \dot{\tilde{z}}\rangle^{2}\right]^{1 / 2},
\end{gathered}
$$

are transverse and axial (longitudinal) beam emittances, respectively. Generally, the emittance varies as the beam propagates. However, as mentioned in the Introduction, we will assume that the nonlinear coupling occurs on a time scale that is shorter than that of the variation of emittance. Hence, we will consider $\epsilon_{\rho}$ and $\epsilon_{z}$ to be constants. In order to calculate the self-field contribution to the envelope equations [second term of the right side of both Eqs. (3a) and (3b)], we need to specify the beam density. For the sake of simplicity, we assume that the distribution remains uniform inside the ellipsoid as the beam propagates. Namely,

$$
n(\mathbf{r}, s)=\frac{3 N}{4 \pi \rho_{b}^{2} z_{b}}, \quad \text { if } \frac{\rho^{2}}{\rho_{b}^{2}}+\frac{z^{2}}{z_{b}^{2}} \leq 1,
$$

and $n=0$ outside the ellipsoid. If we use this density in Eq. (2), the self-field potential inside the ellipsoid becomes ${ }^{38}$

$$
\begin{aligned}
\psi=\frac{3 K}{4} \int_{0}^{\infty} \frac{d u}{\left(\rho_{b}^{2}+u\right)\left(z_{b}^{2}+u\right)^{1 / 2}} \\
\times\left(1-\frac{\rho^{2}}{\rho_{b}^{2}+u}-\frac{z^{2}}{z_{b}^{2}+u}\right) .
\end{aligned}
$$

Substituting Eqs. (5) and (6) in Eq. (3), one obtains a set of coupled equations for the envelope dynamics given by

$$
\begin{aligned}
\frac{d^{2} \rho_{b}}{d s^{2}} & =-k_{\rho}^{2} \rho_{b}+\frac{3 K}{2} h_{\rho}\left(\rho_{b}, z_{b}\right)+\frac{\epsilon_{\rho}^{2}}{\rho_{b}^{3}}, \\
\frac{d^{2} z_{b}}{d s^{2}} & =-k_{z}^{2} z_{b}+3 K h_{z}\left(\rho_{b}, z_{b}\right)+\frac{\epsilon_{z}^{2}}{z_{b}^{3}},
\end{aligned}
$$

where

$$
\begin{aligned}
& h_{\rho}(\rho, z)=\left[\frac{\rho \arccos \left(\frac{z}{\rho}\right)}{\left(\rho^{2}-z^{2}\right)^{3 / 2}}-\frac{z}{\rho\left(\rho^{2}-z^{2}\right)}\right], \\
& h_{z}(\rho, z)=\left[\frac{1}{\left(\rho^{2}-z^{2}\right)}-\frac{z \arccos \left(\frac{z}{\rho}\right)}{\left(\rho^{2}-z^{2}\right)^{3 / 2}}\right] .
\end{aligned}
$$


We can reduce the number of parameters on which the model depends by conveniently scaling $\rho_{b}, z_{b}$, and $s$ to $\left(\epsilon_{\rho} / k_{\rho}\right)^{1 / 2},\left(\epsilon_{\rho} / k_{\rho}\right)^{1 / 2}$, and $k_{\rho}^{-1}$, respectively. The envelope equations (7) then become

$$
\begin{aligned}
& \frac{d^{2} \rho_{b}}{d s^{2}}=-\rho_{b}+\frac{3 \zeta}{2} h_{\rho}\left(\rho_{b}, z_{b}\right)+\frac{1}{\rho_{b}^{3}}, \\
& \frac{d^{2} z_{b}}{d s^{2}}=-\alpha z_{b}+3 \zeta h_{z}\left(\rho_{b}, z_{b}\right)+\frac{\beta^{2}}{z_{b}^{3}},
\end{aligned}
$$

where $\alpha=k_{z}^{2} / k_{\rho}^{2}$ and $\beta=\epsilon_{z} / \epsilon_{\rho}$ are the ratios between the axial and transverse vacuum phase advances and of emittances, respectively, and $\zeta=K /\left(k_{\rho} \epsilon_{\rho}^{3}\right)^{1 / 2}$ is a dimensionless parameter that measures the beam intensity. It is interesting to note that the envelope equations (9) can be derived from a Hamiltonian

$$
\begin{aligned}
H\left(p_{\rho_{b}}, p_{z_{b}}, \rho_{b}, z_{b}\right)= & \frac{p_{\rho_{b}}^{2}}{4}+\frac{p_{z_{b}}^{2}}{2}+\rho_{b}^{2}+\alpha \frac{z_{b}^{2}}{2} \\
& +\frac{1}{\rho_{b}^{2}}+\frac{\beta^{2}}{2 z_{b}^{2}}+3 \zeta \frac{\arccos \left(\frac{z_{b}}{\rho_{b}}\right)}{\sqrt{\rho_{b}^{2}-z_{b}^{2}}},
\end{aligned}
$$

where $p_{\rho_{b}}$ and $p_{z_{b}}$ play the role of the conjugate momenta such that to $d \rho_{b} / d s=\partial H / \partial p_{\rho_{b}}, d p_{\rho_{b}} / d s=-\partial H / \partial \rho_{b}, d z_{b} / d s$ $=\partial H / \partial p_{z_{b}}, d p_{z_{b}} / d s=-\partial H / \partial z_{b}$. Since the Hamiltonian does not explicitly depend on time, $s$, it is a constant of motion along the envelope evolution. The identification of the conserved quantity $H\left(p_{\rho_{b}}, p_{z_{b}}, \rho_{b}, z_{b}\right)$ is important since it allows us to construct Poincaré plots which are a very useful tool for studying the nonlinear dynamics. ${ }^{36}$

\section{SPHERICAL BEAM}

Let us first consider the particular case where the external focusing is isotropic along the transverse and longitudinal directions, and the beam has spherical symmetry. This is a simplifying assumption but may well describe some systems of practical relevance. ${ }^{39}$ It corresponds to taking $\alpha=1$, $\beta=1$, and $\rho_{b}=z_{b}=r_{b}$, where $r_{b}$ is the envelope radius of a spherical beam. Taking the proper limit of Eq. (9), we find that the evolution of the beam envelope is given by

$$
\frac{d^{2} r_{b}}{d s^{2}}=-r_{b}+\frac{\zeta}{r_{b}^{2}}+\frac{1}{r_{b}^{3}} .
$$

A matched solution corresponds to a perfect balance between the focusing and defocusing forces which act on the beam as it propagates so that $r_{b}(s)=r_{b m}=$ const. Equating $d^{2} r_{b} / d s^{2}=0$ in Eq. (11), we find that the matched radius satisfies

$$
r_{b m}-\frac{\zeta}{r_{b m}^{2}}-\frac{1}{r_{b m}^{3}}=0 .
$$

For any given value of the parameter $\zeta$, the above equation presents only one physical solution for $r_{b m}$. If we consider a matched beam with a uniform distribution and substitute the corresponding self-field potential (2) in Eq. (1), we observe that a given particle that stays inside the beam will have motion equation given by $d \mathbf{r} / d s^{2}=-\nu^{2} \mathbf{r}$, in the normalized variables, where $\nu=r_{b m}^{-2}$ is the tune depression. We see that the tune depression and the dimensionless parameter $\zeta$ have a one to one relation and are both quantities that are used as a measure of the space charge intensity in beams. We choose to base our discussions on the parameter $\zeta$. Despite the existence of a matched solution, in practice, the initial beam size may largely vary from the matched so that $r_{b}(0)=\mu r_{b m}$, where $\mu$ is a dimensionless parameter that measures the mismatch amplitude. The beam will then present a breathing mode with its envelope oscillating according to Eq. (11). Furthermore, one can also expect the launched beam to present small asymmetries in its shape, such that $\rho_{b}$ and $z_{b}$ are not exactly the same. Given that these quantities are nonlinearly coupled by the space charge forces, the asymmetry may grow at the expense of the breathing oscillations. In fact, this is the case for unbunched beams, for which it was found that the asymmetric mode can become unstable for mismatches of the order of $100 \%$ (Ref. 33) $(\mu \approx 2)$, which is a realistic value in some applications. ${ }^{40}$ Our first aim here is to determine if and when an analogous symmetry breaking instability occurs for bunched beams.

We begin our investigation by analyzing the envelope phase space described by the Hamiltonian $H\left(p_{\rho_{b}}, p_{z_{b}}, \rho_{b}, z_{b}\right)$ of Eq. (10). Because this is a two-degrees-of-freedom system, Poincaré plots are a useful tool. ${ }^{36,41}$ Here, we choose to plot $\rho_{b}$ vs. $p_{\rho_{b}}$ each time $z_{b}$ hits a local maximum. In the Poincaré plot, all the trajectories must have the same value of the Hamiltonian. Therefore, for a given set of parameters $\zeta$ and $\mu$, we evaluate the corresponding value for the spherical mismatched beam as given by $H\left(0,0, \mu r_{b m}, \mu r_{b m}\right)$ and determine a group of initial conditions that lead to the same $H\left(p_{\rho_{b}}, p_{z_{b}}, \rho_{b}, z_{b}\right)$. In Fig. 1(a), we show the Poincaré plot obtained for $\zeta=1$ and $\mu=2$. We should emphasize that each trajectory in the Poincaré plot corresponds to a different mismatch along the longitudinal and transverse directions, but such that all belong to the same set with $H\left(p_{\rho_{b}}, p_{z_{b}}, \rho_{b}, z_{b}\right)=$ $H\left(0,0, \mu r_{b m}, \mu r_{b m}\right)$ for a single value of parameter $\mu$. We notice the presence of a single stable (elliptic) fixed point at $\rho_{b} \approx 2.44$ and $p_{\rho_{b}}=0$. This point corresponds to a spherical beam breathing mode for which $z_{b}(s)$ and $\rho_{b}(s)$ oscillate at the same frequency. This should not be confused with the matched solution $z_{b}=\rho_{b}=r_{b m}$, for which the envelope does not evolve in time. For all the other trajectories shown in the plot, there is an incommensurate relation between the longitudinal and the transverse frequencies leading to KAM curves that circulate around the fixed point. The fact that the fixed point is stable means that any small asymmetry will not grow along the propagation, and the beam will maintain its nearly spherical shape.

As the parameters are varied, however, the fixed point may loose its stability and become hyperbolic. In this case, the energy of the breathing mode oscillation will be exchanged with the asymmetric mode, with the beam developing an ellipsoidal shape. In order to explore this possibility, we take advantage of the nonlinear dynamical techniques and evaluate the stability index $\Lambda$ of a spherically symmetric breathing oscillation, i.e., of the fixed point of the 

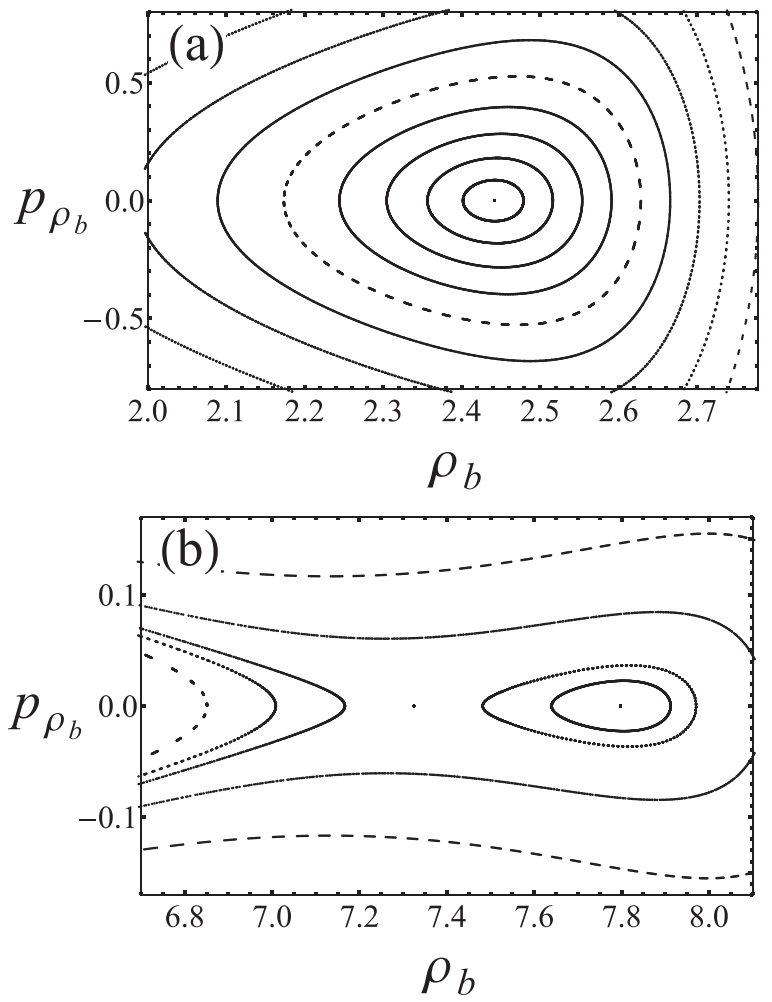

FIG. 1. Poincaré plot of the envelope dynamics in Eq. (9) for 2 different values of the mismatch parameter: (a) $\mu=2$ and (b) $\mu=6$. The remaining parameters are $\alpha=1, \beta=1$, and $\zeta=1$.

corresponding Poincaré plot. We define $\Lambda=\cos \left(\phi_{\text {fix }}\right)$, where $\phi_{\text {fix }}$ is the phase that a trajectory corresponding to a small perturbation around the fixed point advances between two consecutive plots obtained by integrating Eq. (9). Both the location of the fixed point and its stability index are numerically calculated using a Newton-Raphson method. ${ }^{3,37}$ If the fixed point is elliptic, then $\phi_{\text {fix }}$ is real and $|\Lambda|<1$. On the other hand, if the point is hyperbolic, $\phi_{\text {fix }}$ becomes imaginary-trajectories move away exponentially from the point-and $|\Lambda|>1$. Note that the bifurcation (transition between stability and instability) occurs when $\phi_{\text {fix }}$ is a multiple of $\pi$, which means that there is a resonance between the plotting frequency and the fixed point rotating frequency. In Fig. 2, we show the results obtained for $\Lambda$ as the mismatch amplitude is varied for $\zeta=1$. We see that $\Lambda$ increases monotonically with $\mu$. For small mismatches, the fixed point is stable with $|\Lambda|<1$. Approximately at $\mu=5$, the stability index crosses the $\Lambda=1$ line and the fixed point becomes unstable. This can be verified in the Poincaré Plot of Fig. 1(b) which shows the vicinity of the now unstable fixed point located at $\rho_{b} \approx 7.35$ and $p_{\rho_{b}}=0$ that corresponds to the spherically symmetric breathing mode. It is neighbored by two resonant islands, a smaller one to the right and a larger one to the left (just partially shown due to its size). We have performed the stability analysis for other values of the intensity parameter $\zeta$ and found that the symmetry breaking always occurs for $\mu \geq 5$. Therefore, this effect is much less pronounced for 3D spherical beams than for 2D continuous beams, for which the mismatch amplitudes of $\mu \approx 2$ were sufficient to induce symmetry breaking. ${ }^{33}$ A closer inspection of Fig. 2 may give

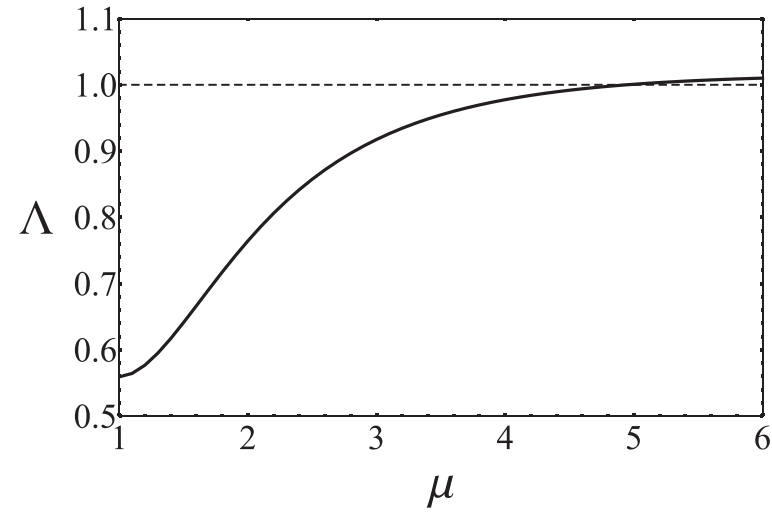

FIG. 2. Stability index $\Lambda$ as a function of the mismatch amplitude $\mu$ for the spherically symmetric breathing mode of oscillation. Only for a mismatch of the order $\mu \approx 5$, this mode becomes unstable. The space-charge parameter used is $\zeta=1$.

us a hint of why such large mismatch amplitudes are necessary in the case of bunched beams. In particular, we note that when $\mu \rightarrow 1$ the stability index is rather far from the bifurcation values $\Lambda= \pm 1$, meaning that the linear oscillating frequencies for the matched solution are also far from being commensurate. Hence, a large oscillating amplitude is necessary in order to lock the frequencies and to generate an instability.

\section{ELLIPSOIDAL BEAM}

In Section III, we investigated a particular case where both focusing forces and emittances are the same along the transverse and longitudinal directions, such that the equilibrium corresponds to a spherical beam of radius $r_{b m}$. However, in many applications, these quantities are different and the equilibrium beam will have an ellipsoidal shape with varying ratios between transverse and longitudinal dimensions. The matched envelopes $\rho_{b m}$ and $z_{b m}$ are determined from the stationary solutions of Eq. (9), leading to

$$
\begin{aligned}
& 2 \rho_{b m}^{4}-3 \zeta \rho_{b m}^{3} h_{\rho}\left(\rho_{b m}, z_{b m}\right)-2=0, \\
& \alpha z_{b m}^{4}-3 \zeta z_{b m}^{3} h_{z}\left(\rho_{b m}, z_{b m}\right)-\beta^{2}=0,
\end{aligned}
$$

which must be solved numerically as a function of the dimensionless parameter $\alpha, \beta$, and $\zeta$. Analogous to the spherical case, by looking at the force of a given particle that stays inside a matched ellipsoidal beam with uniform density, we can define tune depression parameters, which read $\nu_{\rho}=\rho_{b m}^{-2}$ and $\nu_{z}=\beta z_{b m}^{-2} \alpha^{-1 / 2}$ for the transverse and longitudinal directions, respectively. We note that the tune depressions are completely determined once the parameters $\alpha, \beta$, and $\zeta$ are given. We choose to base our discussions on the latter set of parameters. Clearly, in such cases, there is no point of discussing symmetry breaking, since the matched beam is already asymmetric. Nevertheless, nonlinear effects can still be relevant as they can cause a strong coupling and energy exchange between oscillations in the different directions. ${ }^{35}$ In fact, differently from the spherical case of Sec. III where large oscillating amplitudes were found to be necessary to induce frequency lock and instability, by varying the 

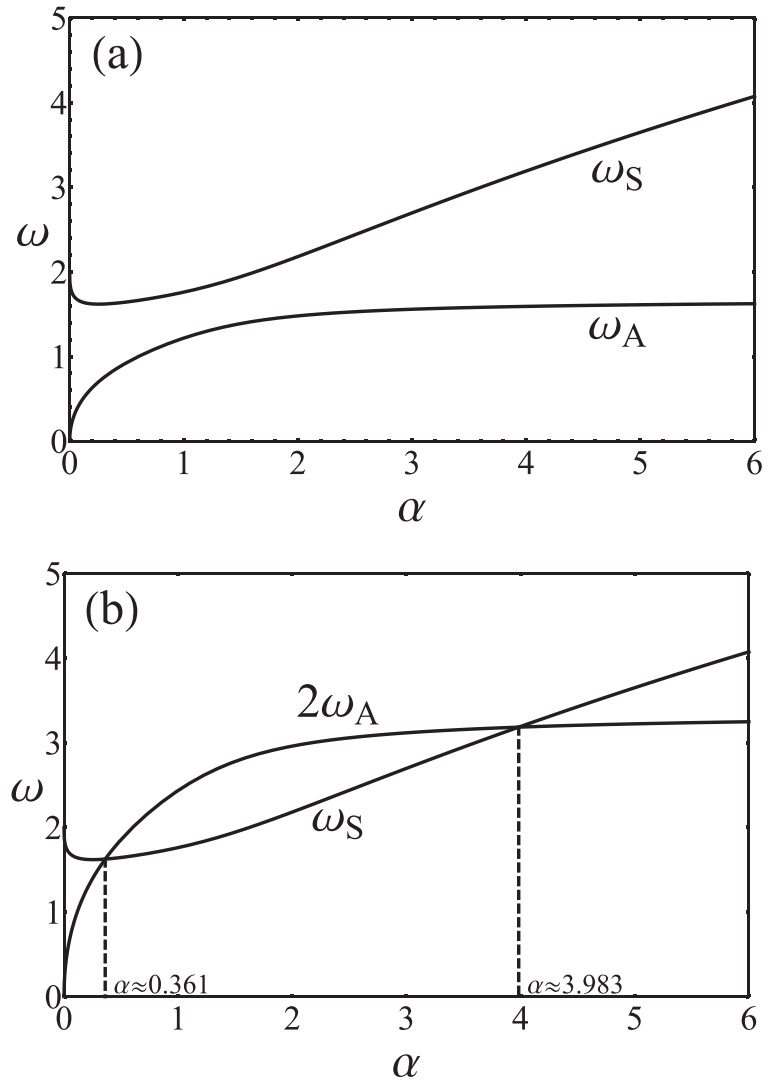

FIG. 3. Dispersion relation for the linearized envelope equations as a function of $\alpha \equiv k_{z}^{2} / k_{\rho}^{2}$. In (a), the solid curves for $\omega_{S}$ and $\omega_{A}$ represent the symmetric and antisymmetric eigenfrequencies, respectively, for $\beta=1$ and $\zeta=5$. In (b), we plot $2 \omega_{A}$ to show the $1: 2$ resonance conditions which are $\alpha \approx 0.361$ and $\alpha \approx 3.983$ for the same parameters.

parameters $\alpha$ and $\beta$, one may find certain conditions for which even small amplitude mismatch can lead to an instability.

To verify this, we look at the linear stability of Eq. (9) around the matched solution of Eq. (13). Substituting $\rho_{b}=$ $\rho_{b m}+\delta \rho_{b} e^{i \omega t}$ and $z_{b}=z_{b m}+\delta z_{b} e^{i \omega t}$ in Eq. (9) and retaining only the linear terms in $\delta \rho_{b}$ and $\delta z_{b}$, we obtain equations for the eigenfrequencies $\omega$.

We find two pairs of solutions: $\pm \omega_{S}$ associated with a symmetric mode where $\delta \rho_{b}$ and $\delta z_{b}$ oscillate in phase and $\pm \omega_{A}$ associated with an antisymmetric mode where $\delta \rho_{b}$ and $\delta z_{b}$ oscillate in opposite phases. In Fig. 3(a), we show the solutions of the dispersion relation for $\beta=1, \zeta=5$, and varying $\alpha \equiv k_{z}^{2} / k_{\rho}^{2}$. We notice that the branches of the symmetric and antisymmetric modes never touch each other; therefore, there is no value of $\alpha$ for which there is a direct $1: 1$ resonance between these modes. While this was shown for just one set of parameters, we find that the same is true for any value of $\alpha, \beta$, and $\zeta$. Hence the system is always linearly stable, as was also predicted in Ref. 42. However, as shown in Fig. 3(b), there is a 1:2 resonance between the symmetric and antisymmetric modes for two values of $\alpha$-one for $\alpha<1$ and the other for $\alpha>1$. Near where this condition is met, one may expect a strong nonlinear coupling between the different dynamical degrees of freedom.

To investigate this issue and to see how finite amplitude mismatches affect the nonlinear envelope dynamics, we
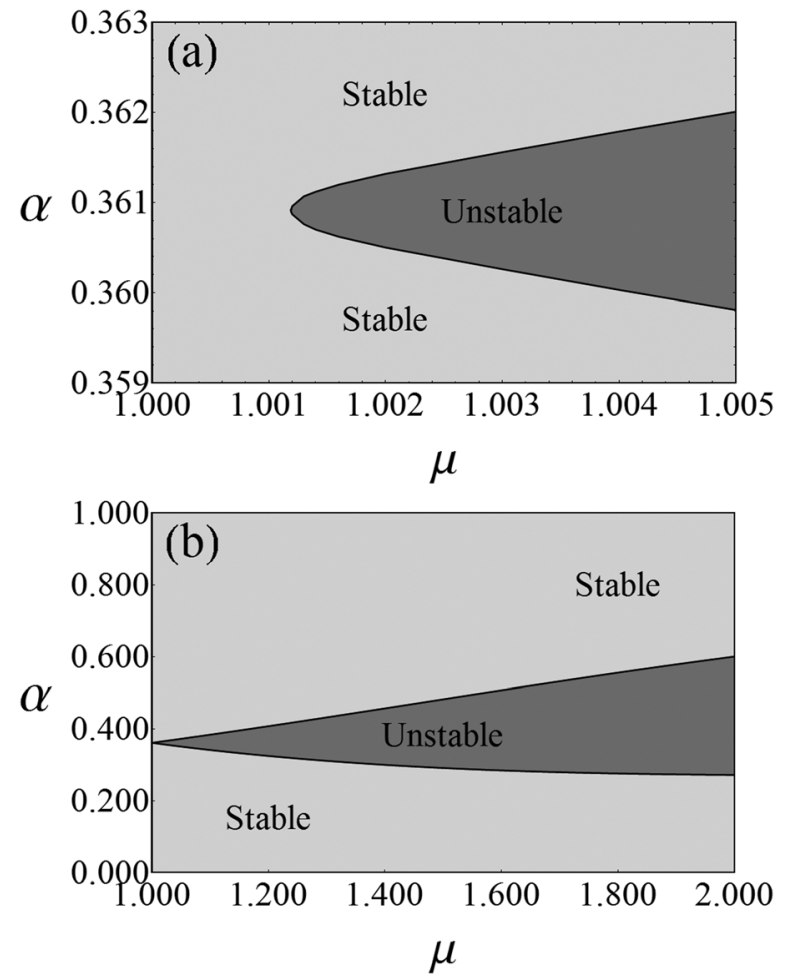

FIG. 4. Stability diagram of the mismatch $\mu$ vs. $\alpha$. The symmetric mode of oscillation is stable in the light gray areas and unstable in the dark gray. This instability is a period-doubling bifurcation of the mode. (a) is a zoom of (b) near $\mu \approx 1$. The remaining parameters are $\beta=1$ and $\zeta=5$.

once again take advantage of the Poincaré plots and stability index. We focus on the situations for which $\alpha<1$, but the analogous results are found for $\alpha>1$. We start by using the Newton-Raphson method to locate and determine the stability index $\Lambda$ of the trajectory for which $\rho_{b}(s)$ and $z_{b}(s)$ oscillate with the same frequency. In Fig. 4, we show stability diagrams for the parameter space $\alpha$ vs. $\mu$. In analogy to the case of spherical beams, for a given mismatch parameter, we consider the trajectories that belong to the set that satisfies $H\left(p_{\rho_{b}}, p_{z_{b}}, \rho_{b}, z_{b}\right)=H\left(0,0, \mu \rho_{b m}, \mu z_{b m}\right)$ for a single value of $\mu$. The remaining parameters are $\beta=1$ and $\zeta=5$. In the diagrams, the light (dark) gray region corresponds to a stable (unstable) solution with $|\Lambda|<1(|\Lambda|>1)$. In panel (a), we show in detail a low mismatch parameter region for $\beta=1$ and $\zeta=5$. We notice that the unstable region does not touch the $\mu=1$ axis - as expected from the linear stability analysis. But, it gets very close to it near $\alpha=0.361$ where the $2: 1$ resonance condition is satisfied and mismatch amplitudes as small as $0.1 \%$ are sufficient to drive the mode unstable. In fact, it is worth noting that differently from the spherical case of Sec. III, in the ellipsoidal case, the bifurcation occurs for $\Lambda=-1$, characterizing a period-doubling bifurcation typical of $2: 1$ resonances. $^{37}$ As the mismatch amplitude is increased, the region of instability gets wider and more values of $\alpha$ are affected, as can be seen in Fig. 4(b).

To further analyze the nonlinear stability and its effect on the envelope dynamics, we now investigate the phase space using Poincaré plots. We construct these in the same way as was explained in Sec. III, with the exception that we choose to plot $z_{b}$ vs. $p_{z_{b}}$ every time that $\rho_{b}$ hits a maximum. 

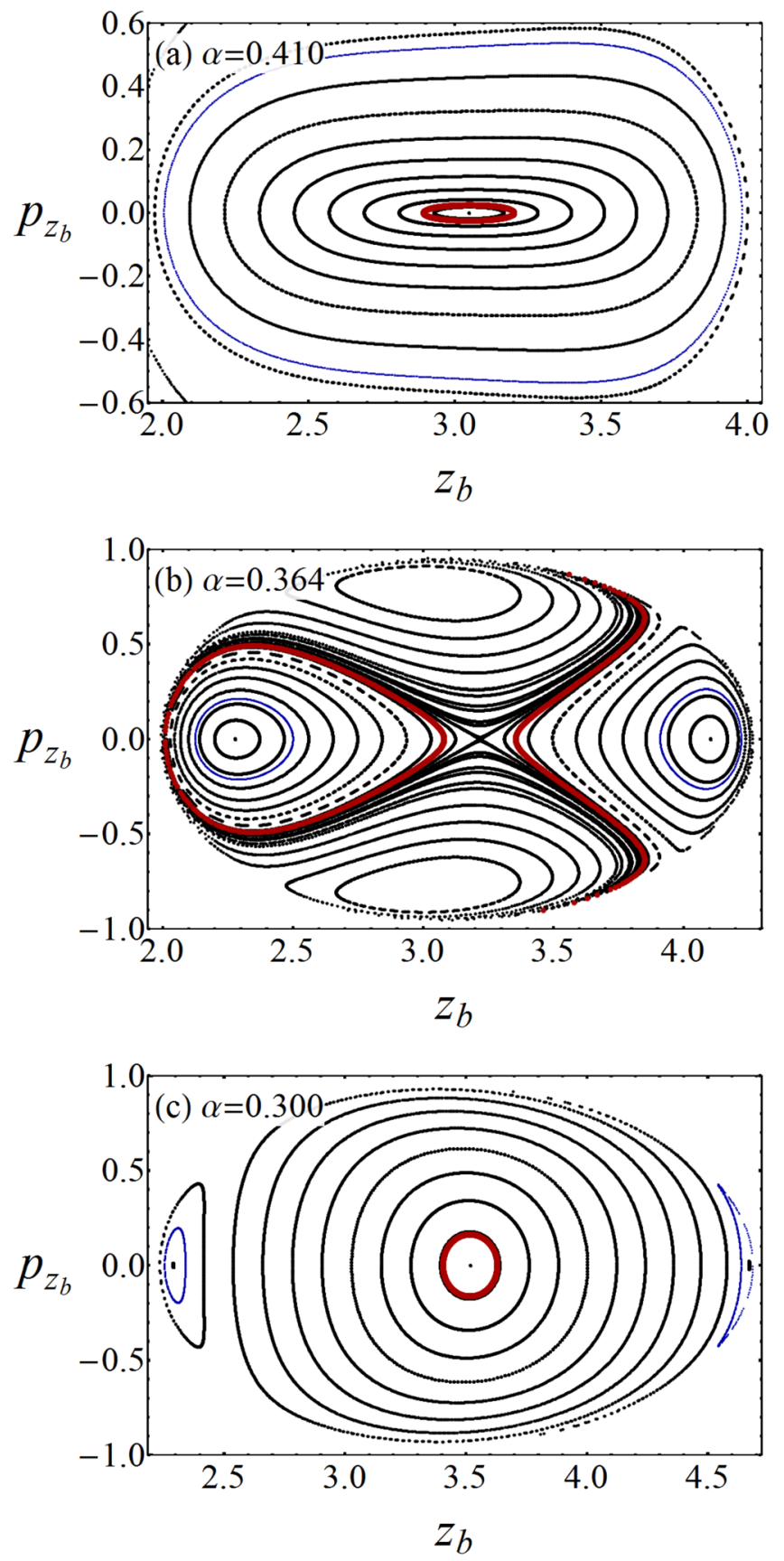

FIG. 5. Poincaré plot of the envelope dynamics in Eq. (9). We plot $z_{b}$ vs. $p_{z_{b}}$ at each maximum of $\rho_{b}$. In (a), for $\alpha=0.41$, the breathing mode is stable. In (b), for $\alpha=0.364$, the fixed point that represents this mode becomes unstable via period doubling bifurcation. In (c), for $\alpha=0.3$, the breathing mode becomes stable once again. The remaining parameters are $\beta=1, \zeta=5$, and $\mu=1.2$.

This is done for convenience to make the plot features more apparent. Figure 5 shows the plots obtained for $\beta=1, \zeta=5$, and $\mu=1.2$ for three different values of $\alpha$. For the sake of illustration, we highlight two distinct initial conditions in the Poincaré plot. The red one represents the case where $z_{b}(0)=$ $z_{b m}$ and all the initial mismatch is placed in $\rho_{b}$, whereas the blue is the opposite in the sense that $\rho_{b}(0)=\rho_{b m}$ and all the initial mismatch is in $z_{b}$. In panel (a) for $\alpha=0.41$, we notice a stable fixed point at $z_{b} \approx 3.05$ and $p_{z_{b}}=0$. This point corresponds to the trajectory for which $\rho(s)$ and $z(s)$ oscillate with the same frequency and whose stability is given in Fig. 4(b). The red trajectory is close to the fixed point and presents a small amplitude oscillation because most of the oscillation energy is in $\rho_{b}$. On the other hand, the blue one is far from the fixed point and already presents a large amplitude oscillation with a large variation between $z_{\text {bmin }} \approx 2.0$ and $z_{\text {bmax }} \approx 4$.0. In panel (b), for $\alpha=0.364$, inside the unstable region of Fig. 4(b), the fixed point at $z_{b} \approx 3.22$ and $p_{z_{b}}=$ 0 suffers a period-doubling bifurcation and becomes hyperbolic with the appearance of two neighboring resonant islands. Initial conditions near the fixed point are heavily affected by the bifurcation and largely increase their oscillation amplitudes. In particular, note that for the red trajectory, the difference between $z_{\text {bmax }}$ and $z_{\text {bmin }}$ increases significantly compared to the stable case of panel (a).

This means that there is a gain of energy for the longitudinal envelope $z_{b}$, which must come at the expense of a decrease of energy of the transverse envelope $\rho_{b}$. This shows a strong coupling between the modes governed by the unstable fixed point. Note, however, that the same is not true for the blue trajectory whose amplitude of variation, $z_{b \max }-z_{b \min }$, has not changed much, as compared to panel (a). For completeness, we show in panel (c), for $\alpha=0.3$, what happens if we further decrease the value of $\alpha$ until it reaches the lower stable region of Fig. 4(b). We note that the two islands that arise from the period-doubling bifurcation are still present, but between them the fixed point, located at $z_{b} \approx 3.52$ and $p_{z_{b}}=0$, becomes stable once again. The variation $z_{b \max }$ $z_{b \min }$ for the red curve near the fixed point decreases to values similar to those found in panel (a), whereas for the blue curve it remains nearly the same large value as those found in panels (a) and (b). Hence, overall we notice that the bifurcations of the fixed point can lead to big changes in the envelope dynamics. These changes tend to affect much more certain mismatched solutions than others. Poincaré plots are found to be a useful tool to discriminate those trajectories that are more or less affected. We have also investigated the Poincaré plots for the $\rho_{b}$ vs. $p_{\rho_{b}}$ phase-space and have found that the results agree with the ones discussed above.

\section{SELF-CONSISTENT NUMERICAL SIMULATIONS}

In the derivation of the envelope equations, two major assumptions were used, namely, that the emittance is preserved and that the particle density remains uniform and ellipsoidal throughout the transport. In order to test the validity of these assumptions and to verify the results obtained in Section IV regarding the role of nonlinear stability in the beam transport, we have also performed molecular dynamics simulations.

Taking advantage of the azimuthal symmetry of the particle distribution, in the simulation, we describe the beam as formed by a series of concentric charged rings of different radii. This dramatically decreases the computation time compared to explicit point particle simulations. The potential at a position $(x, y, z)$ generated by a charged ring of radius $\rho_{i}$, charge $K / N_{R}$, located at $z_{i}$ and centered on the $z$-axis is given by ${ }^{43}$ 


$$
\begin{aligned}
\psi_{i}(\rho, z)= & \frac{2}{\pi} \frac{\left(K / N_{R}\right)}{\sqrt{\left(\rho+\rho_{i}\right)^{2}+\left(z-z_{i}\right)^{2}}} \\
& \times \bar{K}\left(\frac{4 \rho \rho_{i}}{\left(\rho+\rho_{i}\right)^{2}+\left(z-z_{i}\right)^{2}}\right)
\end{aligned}
$$

where $\rho=\sqrt{x^{2}+y^{2}}, N_{R}$ is the number of rings in the simulation, and $\bar{K}(m)$ is the complete elliptic integral of the first kind. Hence, the radial and axial evolution of each ring are given by

$$
\begin{gathered}
\frac{d^{2} \rho_{i}}{d s^{2}}=-k_{\rho}^{2} \rho_{i}+\frac{p_{\varphi}^{2}}{\rho_{i}^{3}}-\sum_{j=1, j \neq i}^{N_{R}} \frac{\partial \psi_{j}\left(\rho_{i}, z_{i}\right)}{\partial \rho_{i}}, \\
\frac{d^{2} z_{i}}{d s^{2}}=-k_{z}^{2} z_{i}-\sum_{j=1, j \neq i}^{N_{R}} \frac{\partial \psi_{j}\left(\rho_{i}, z_{i}\right)}{\partial z_{i}},
\end{gathered}
$$

where $p_{\varphi}$ is the angular momentum associated with the azimuthal coordinate, which is a conserved quantity.

In the simulations, we used $N_{R}=4225$ rings, which was found to be sufficient to guarantee the convergence of results. The rings were uniformly distributed inside an ellipsoid of semi-axis radius $\rho_{b}$ and $z_{b}$. In analogy with a KV distribution for continuous beams, ${ }^{44}$ the velocity distribution
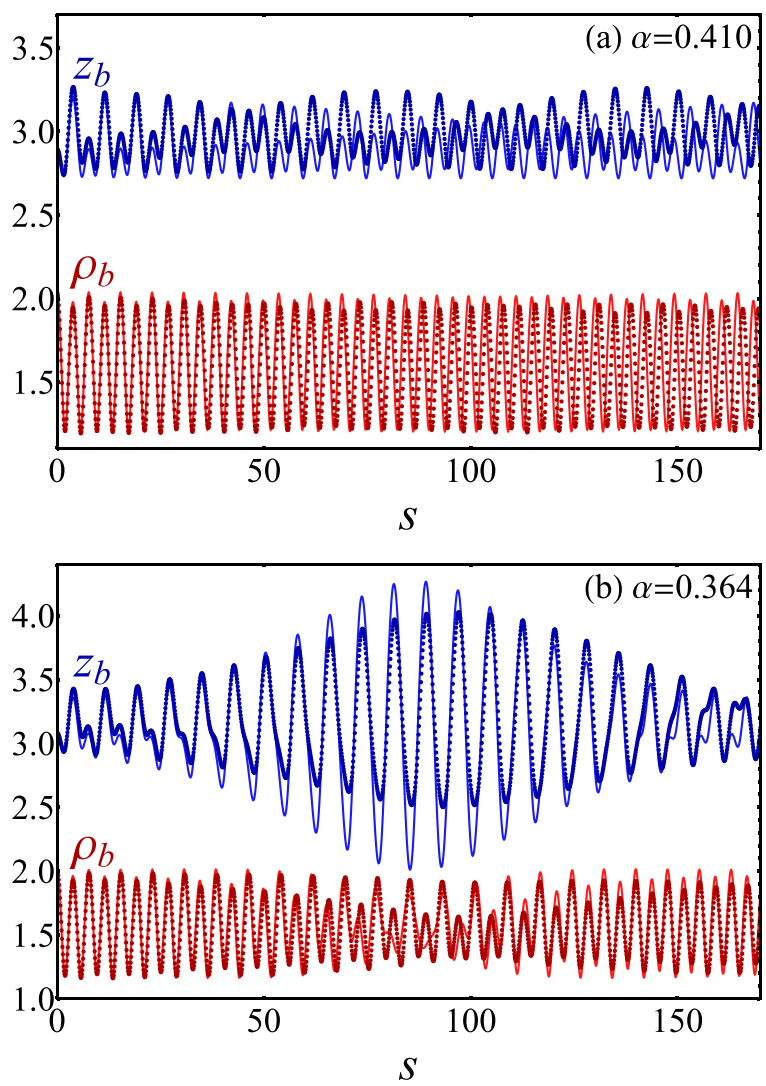

FIG. 6. Comparison between a molecular dynamics simulation and the envelope model from Eq. (9). We see a general good agreement across the board. While in (a), for $\alpha=0.41$, which is a stable situation, there is almost no coupling between the degrees-of-freedom; in (b), for $\alpha=0.364$, the instability generates a strong coupling and a large exchange of energy between $\rho_{b}$ and $z_{b}$. The remaining parameters are $\beta=1, \zeta=5$, and $\mu=1.2$, and the initial condition in both cases is $z_{b}(0)=z_{b m}$. was chosen to have a parabolic dependence on the temperature with the distance from the beam center, with a vanishing velocity dispersion at the beam boundary.

In Fig. 6, we compare the envelope evolution predicted by Eq. (9) (solid curves) against the RMS sizes obtained from the simulations (symbols). We choose the same parameters as in Fig. 5, namely, $\beta=1, \zeta=5$, and $\mu=1.2$. In Fig. $6(a)$, we show the results for $\alpha=0.41$ and an initial condition with $z_{b}(0)=z_{b m}$, such that all the mismatch is in the transverse direction-this corresponds to the red trajectory of Fig. 5(a). It is clear from Fig. 6(a) that the coupling between $\rho_{b}$ and $z_{b}$ is weak, since there is little or no exchange of energy between their oscillations. We see that the results from the simulation for the transverse size $\rho_{b}$ are very accurately described by the envelope model. For the longitudinal direction $z_{b}$, we also find a good agreement, particularly regarding the amplitude of oscillation.

In Fig. 6(b), we show the results for an unstable case with $\alpha=0.364$, with the same parameters as in Fig. 5(b). Again, we consider the solution for which $z_{b}(0)=z_{b m}$ and all the mismatch is in the transverse direction-analogous to the red curve in Fig. 5(b). We clearly see the exchange of energy between the degrees-of-freedom, as the amplitude of $z_{b}$ increases significantly, reaching its pick at $s \approx 80$, at expense of a decrease of the oscillation amplitude of $\rho_{b}$. The comparison with simulation shows again an excellent agreement for
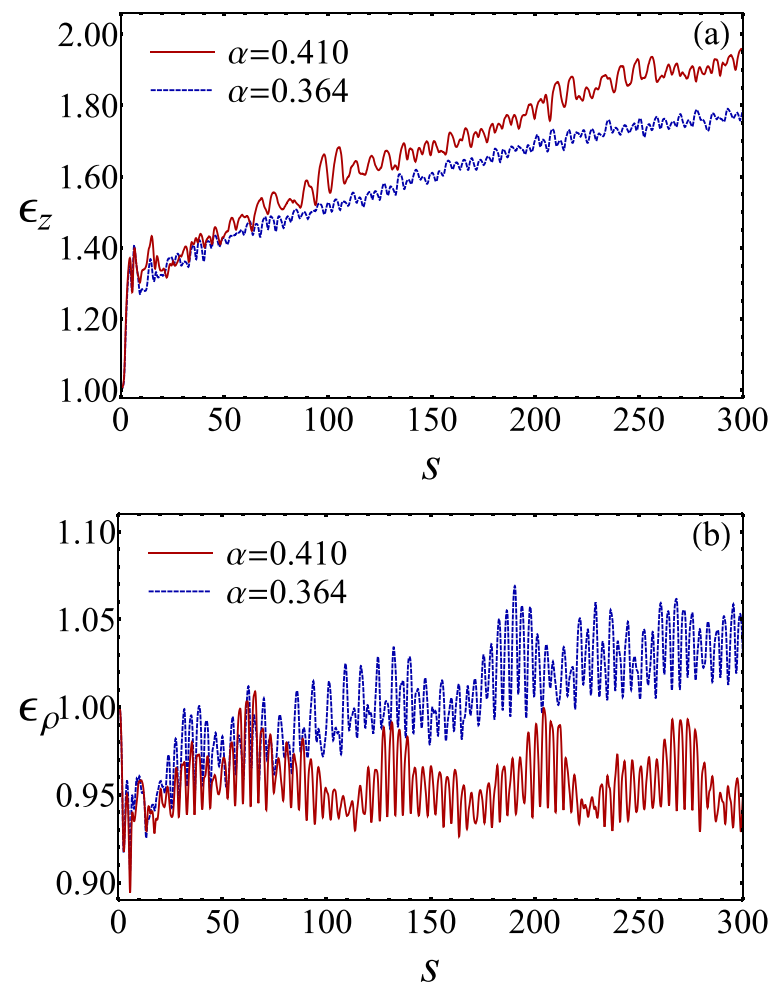

FIG. 7. Emittance as a function of the normalized time obtained from the simulation. The initial emittance in both longitudinal and transverse directions at $s=0$ is normalized to one. In panel (a), we show the longitudinal emittance, which behaves very similarly in both stable and unstable situations. However, the transverse emittance, shown in panel (b), presents a distinct behavior: for the stable case, solid red curve, the emittance oscillates around a fixed value; for the unstable case, dashed blue curve, there is an increase in the emittance. The parameters are the same as in Fig. 6. 
$\rho_{b}$. For $z_{b}$, we see that the behavior of the complete system is well described by the envelope equation, even though the increase of the amplitude caused by the instability is not as large as predicted by the model.

Since in the molecular dynamics simulations the emittance evolves self-consistently, we can use it to investigate how the envelope instability may affect the emittance dynamics. Particularly, we consider the stable and unstable cases of Figs. 6(a) and 6(b), respectively. Since the beams are mismatched, it is natural to expect that the emittance will grow as the system evolves. In fact, we find in the simulations that the longitudinal emittance presents a steady increase which is similar in both stable and unstable cases, being just a little slower in the latter case, as shown in Fig. 7(a). However, the transverse emittance presents a very distinct and somewhat unexpected behavior, as shown in Fig. 7(b). Although all the initial mismatch is imposed on $\rho_{b}$, the transverse emittance shows a rapid decrease at the start of the evolution. This is probably caused by a quick particle redistribution from its uniform initial condition. From there on, the instability seems to have an important role. While for the stable case (red solid line) there is no emittance growth with $\epsilon_{\rho}$ just oscillating around a fixed average which is smaller than its initial value, for the unstable case (dashed blue line) there is a clear increase of $\epsilon_{\rho}$. This increase is an indication of the effective coupling between longitudinal and transverse degrees of freedom and explains the slower growth of $\epsilon_{z}$ seen for the unstable case in Fig. 7(a).

\section{CONCLUSION}

In this paper, we have investigated the nonlinear coupling between the envelope modes of oscillation of intense bunched beams. In contrast to previous investigations which consider a perturbative analysis to determine the linear stability of beams with vanishingly small mismatches, $1,3,5-8,45$ here, we adopted a nonperturbative analysis of the envelope equations which takes into account finite mismatches of arbitrary amplitude. We first considered a case of spherically symmetric beams and investigated how breathing mode oscillations may nonlinearly induce a growth of ellipsoidal modes. It was found that above a certain mismatch amplitude there is an effective exchange of energy between the modes which results in beam symmetry breaking. In comparison with continuous beams, ${ }^{33}$ however, we find that for bunched beams the nonlinear instability only takes place for much larger mismatches. We then considered a more general case in which the focusing forces along the longitudinal and transverse directions are not the same, such that the matched beam already presents an ellipsoidal shape. In this case, we found that depending on the parameters of the system even a very small mismatch amplitude can already drive an instability which effectively couples longitudinal and transversal envelope oscillations. Using Poincaré plots, we identified the instability as a period doubling bifurcation and determined which orbits are most affected by the instability. Evaluating the stability index, we constructed a phase diagram showing where the instability will occur. Molecular dynamics simulations were performed to verify the onset of the nonlinear instability and to demonstrate its effect on the evolution of the RMS size and on emittance of the beam.

\section{ACKNOWLEDGMENTS}

This work was partially supported by CNPq, FAPERGS, and INCT-FCx, Brazil, and by the US-AFOSR under Grant No. FA9550-16-1-0280.

${ }^{1}$ J. Struckmeier and M. Reiser, Part. Accel. 14, 227 (1984).

${ }^{2}$ C. Chen and R. C. Davidson, Phys. Rev. Lett. 72, 2195 (1994).

${ }^{3}$ R. Pakter and F. B. Rizzato, Phys. Rev. Lett. 87, 044801 (2001).

${ }^{4}$ R. Pakter and F. B. Rizzato, Phys. Rev. E 65, 056503 (2002).

${ }^{5}$ S. M. Lund and B. Bukh, Phys. Rev. Spec. Top. Accel. Beams 7, 024801 (2004).

${ }^{6}$ C. Li and Y. L. Zhao, Phys. Rev. Spec. Top. Accel. Beams 17, 124202 (2014).

${ }^{7}$ C. Li and Q. Qin, Phys. Plasmas 22, 023108 (2015).

${ }^{8}$ C. Li, Z. Liu, Y. Zhao, and Q. Qin, Nucl. Instrum. Methods Phys. Res. A 813, 13 (2016).

${ }^{9}$ C. Chen and R. Pakter, Phys. Plasmas 7, 2203 (2000).

${ }^{10}$ P. A. P. Nghiem, N. Chauvin, M. Comunian, O. Delferrière, R. Duperrier, A. Mosnier, C. Oliver, W. Simeoni, Jr., and D. Uriot, Laser Part. Beams 32, 109 (2014).

${ }^{11}$ M. Reiser, J. Appl. Phys. 70, 1919 (1991).

${ }^{12}$ K. Fiuza, F. B. Rizzato, and R. Pakter, Phys. Plasmas 13, 023101 (2006)

${ }^{13}$ E. G. Souza, A. Endler, F. B. Rizzato, and R. Pakter, Phys. Rev. Lett. 109, 075003 (2012)

${ }^{14}$ R. L. Gluckstern, Phys. Rev. Lett. 73, 1247 (1994).

${ }^{15}$ H. Okamoto and M. Ikegami, Phys. Rev. E 55, 4694 (1997).

${ }^{16}$ T. P. Wangler, K. R. Crandall, R. Ryne, and T. S. Wang, Phys. Rev. Spec. Top. Accel. Beams 1, 084201 (1998).

${ }^{17}$ J. Qiang and R. D. Ryne, Phys. Rev. Spec. Top. Accel. Beams 3, 064201 (2000).

${ }^{18}$ M. Comunian, A. Pisent, A. Bazzani, G. Turchetti, and S. Rambaldi, Phys. Rev. Spec. Top. Accel. Beams 4, 124201 (2001).

${ }^{19}$ C. K. Allen, K. C. D. Chan, P. L. Colestock, K. R. Crandall, R. W. Garnett, J. D. Gilpatrick, W. Lysenko, J. Qiang, J. D. Schneider, M. E. Schulze, R. L. Sheffield, H. V. Smith, and T. P. Wangler, Phys. Rev. Lett. 89, 214802 (2002).

${ }^{20}$ R. P. Nunes, R. Pakter, and F. B. Rizzato, Phys. Plasmas 14, 023104 (2007); J. Appl. Phys. 104, 013302 (2008).

${ }^{21}$ Y. Levin, R. Pakter, and T. N. Teles, Phys. Rev. Lett. 100, 040604 (2008).

${ }^{22}$ M. A. Dorf, R. C. Davidson, and E. A. Startsev, Phys. Plasmas 18, 043109 (2011).

${ }^{23}$ P. A. P. Nghiem, N. Chauvin, W. Simeoni, Jr., and D. Uriot, Appl. Phys. Lett. 104, 074109 (2014).

${ }^{24}$ P. A. P. Nghiem, M. Valette, N. Chauvin, N. Pichoff, and D. Uriot, Phys. Plasmas 22, 083115 (2015).

${ }^{25}$ T. M. Corrêa da Silva, R. Pakter, F. B. Rizzato, and Y. Levin, Phys. Plasmas 22, 023102 (2015).

${ }^{26}$ A. Anderson, Part. Accel. 21, 197 (1987)

${ }^{27}$ S. Bernal, R. A. Kishek, M. Reiser, and I. Haber, Phys. Rev. Lett. 82, 4002 (1999).

${ }^{28}$ S. G. Anderson and J. B. Rosenzweig, Phys. Rev. Spec. Top. Accel. Beams 3, 094201 (2000).

${ }^{29}$ S. M. Lund, D. P. Grote, and R. C. Davidson, Nucl. Instrum. Methods Phys. Res. A 544, 472 (2005).

${ }^{30}$ F. B. Rizzato, R. Pakter, and Y. Levin, Phys. Plasmas 14, 110701 (2007).

${ }^{31}$ R. P. Nunes, R. Pakter, F. B. Rizzato, A. Endler, and E. G. Souza, Phys Plasmas 16, 033107 (2009).

${ }^{32}$ E. G. Souza, A. Endler, R. Pakter, F. B. Rizzato, and R. P. Nunes, Appl. Phys. Lett. 96, 141503 (2010).

${ }^{33}$ W. Simeoni, Jr., F. B. Rizzato, and R. Pakter, Phys. Plasmas 13, 063104 (2006)

${ }^{34}$ R. Pakter, B. Marcos, and Y. Levin, Phys. Rev. Lett. 111, 230603 (2013).

${ }^{35}$ I. Hofmann, J. Qiang, and R. Ryne, in Proceedings of 1999 Particle Accelerator Conference (1999), Vol. 1, p. 137.

${ }^{36}$ A. J. Lichtenberg and M. A. Lieberman, Regular and Stochastic Motion (Springer-Verlag, New York, 1992).

${ }^{37}$ R. Pakter, G. Corso, T. S. Caetano, D. Dillenburg, and F. B. Rizzato, Phys. Plasmas 1, 4099 (1994). 
${ }^{38}$ R. C. Davidson and H. Qin, Physics of Intense Charged Particle Beams in High Energy Accelerators (World Scientific, Singapore, 2001).

${ }^{39}$ P. A. P. Nghiem, N. Chauvin, M. Comunian, O. Delferriere, R. Duperrier, A. Mosnier, C. Oliver, and D. Uriot, Nuclear Instrum. Methods Phys. Res., Sect. A 654, 63 (2011).

${ }^{40}$ R. Pakter and C. Chen, IEEE Trans. Plasma Sci. 28, 502 (2000).
${ }^{41}$ R. Pakter, S. R. Lopes, and R. L. Viana, Physica D 110, 277 (1997).

${ }^{42}$ I. Hofmann, Phys. Rev. E 57, 4713 (1998).

${ }^{43}$ O. Ciftja, A. Babineaux, and N. Hafeez, Eur. J. Phys. 30, 623 (2009).

${ }^{44} \mathrm{I}$. M. Kapchinskij and V. V. Vladimirskij, in Proceedings of the International Conference on High Energy Accelerators (CERN, Geneva, 1959), p. 274.

${ }^{45}$ F. B. Rizzato and R. Pakter, Phys. Rev. Lett. 89, 184102 (2002). 